\title{
THE TEMPERATURE DEPENDENCE OF THE WIDTH OF THE GIANT DIPOLE RESONANCE
}

\author{
W. E. Ormand \\ Dept. of Physics, University of Tennessee \\ Knoxville, TN 37996-1200 \\ and \\ Physics Division, Oak Ridge National Laboratory* \\ Oak Ridge, Tennessee 37831-6373
}

\section{P. F. Bortignon}

Dipartimento di Fisica, Università Degli Studi di Milano and Istituto Nazionale di Fisica Nucleare, Sezione di Milano

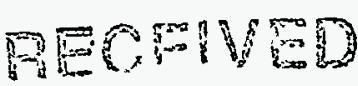

Via Celoria 16, 20133 Milano, Italy

and

\section{R. A. Broglia}

Dipartimento di Fisica, Università Degli Studi di Milano and Istituto Nazionale di Fisica Nucleare, Sezione di Milano

Via Celoria 16, 20133 Milano, Italy

and

Niels Bohr Institute, Blegdamsvej 17

DK-2100 Copenhagen $\varnothing$, Denmark

\author{
to be published in Proceedings of
}

Conference on Giant Resonances
Groningen, The Netherlands

June 28 - July 1, 1995

*Managed by Lockheed Martin Energy Systems under Contract DE-AC05-84OR21400 with the U.S. Department of Energy.

\section{DISCLAIMER}

This report was prepared as an account of work sponsored by an agency of the United States Government. Neither the United States Government nor any agency thereof, nor any of their employees, makes any warranty, express or implied, or assumes any legal liability or responsibility for the accuracy, completeness, or usefulness of any information, apparatus, product, or process disclosed, or represents that its use would not infringe privately owned rights. Reference herein to any specific commercial product, process, or service by trade name, trademark, manufacturer, or otherwise does not necessarily constitute or imply its endorsement, recommendation, or favoring by the United States Government or any agency thereof. The views and opinions of authors expressed herein do not necessarily state or reflect those of the United States Government or any agency thereof.
"The submitted manuscript has been authored by a contractor of the U.S. Government under Contract No. DE-ACOS. $840 R 21400$. Accordingly, the U.S. Government retains a nonexclusive, royaltyfree license to publish or reproduce the published form of this contribution, or allow others to do so, for U.S. Government purposes." 


\section{THE TEMPERATURE DEPENDENCE OF THE WIDTH OF THE GIANT-DIPOLE RESONANCE}

W.E. Ormand $d^{a}$, P.F. Bortignon ${ }^{c}$ and R.A. Broglia ${ }^{c d}$

aPhysics Department, 401 Nielsen Hall, University of Tennessee, Knoxville, TN 37996-1200 USA

bPhysics Division, Oak Ridge National Laboratory, P.O. Box 2008, MS-6373 Building 6003, Oak Ridge, TN 37831-6373 USA

'Dipartimento di Fisica, Università Degli Studi di Milano, and Istituto Nazionale di Fisica Nucleare, Sezione di Milano, Via Celoria 16, 20133 Milano, Italy

${ }^{\mathrm{d}}$ The Niels Bohr Institute, University of Copenhagen, Blegdamsvej 17, DK-2100 Copenhagen $\varnothing$, Denmark

The giant-dipole resonance (GDR) in ${ }^{120} \mathrm{Sn}$ and ${ }^{208} \mathrm{~Pb}$ is studied as a function of excitation energy, angular momentum, and intrinsic width within the context of the adiabatic model. Theoretical evaluations of the full-width-at-half-maximum (FWHM) for the GDR strength function are compared with recent experimental data and are found to be in good agreement.

\section{INTRODUCTION}

The principal objective of many experimental programs has been the study of the properties of the giant-dipole resonance (GDR) at finite temperature [1]. These experiments yield important information about theoretical models of the GDR; in particular, the effects of quantal and thermal fluctuations in the damping of the giant vibration. Currently, the four primary issues are: (1) the temperature dependence of the intrinsic width [2]; (2) the time scale for thermal fluctuations and the validity of either the adiabatic picture [3] or the occurrence of motional narrowing [4]; (3) the existence of a limiting temperature for the observation of collective motion in nuclei [5]; and (4) the influence of the lifetime of the compound nucleus on the observed width of the GDR [6]. Of particular importance to address these issues is a systematic and comprehensive comparison between experiment and theory over a wide range of temperatures for several nuclei.

One of the principal experimental techniques for observing the GDR in hot nuclei has been compound-nuclear reactions induced in heavy-ion collisions [1]. One drawback of compound nuclear reactions to study the GDR at finite temperature is that because of the dynamics of heavy-ion collisions, the compound system is generally formed at high angular momentum. Indeed, those experiments associated with the highest excitation 
energy also have an average angular momentum of approximately $40 \hbar$, with a maximum at approximately $60 \hbar$. As such, it is difficult to separate the effects on the GDR due to large-amplitude thermal fluctuations of the shape from those due to angular momentum.

Recently, two experimental methods for studying the GDR in hot nuclei have been introduced in order to study the effects of excitation energy and angular momentum separately. In experiments involving compound nuclear reactions, large arrays of gamma detectors have been used in order to identify GDR photons associated with a system of a definite angular momentum. By gating on the multiplicity of low-energy $E 2$ photons, the GDR may be studied within an angular momentum window [7]. This window is usually in the range of 10-15 units of angular momentum wide, and centered between 30-50 $\hbar$. An alternative technique is to excite a target nucleus using inelastic alpha scattering [8], which, because of the small mass of the projectile, yields an excited system with a fairly small angular momentum - often less than $20 \%$. Thus, for the first time it is now possible to analyze experimental data for the GDR in hot nuclei in terms of the effects due to large-amplitude thermal fluctuations and angular momentum individually.

In this report, we present a systematic study of the properties of the GDR (in particular the full-width-at-half-maximum (FWHM) of the strength function) as a function of temperature, angular momentum, and intrinsic width for ${ }^{120} \mathrm{Sn}$ and ${ }^{208} \mathrm{~Pb}$ in comparison with recent experimental data from inelastic alpha scattering [8]. Because of the systematic analysis over a range of temperatures and the relatively low angular momentum of the emitting system, it is possible to draw conclusions regarding the roles played by shell corrections, angular momentum, the temperature dependence of the intrinsic width, and the lifetime of the compound nucleus on the observedi width of the GDR.

\section{ADIABATIC MODEL FOR THE GDR}

Under the assumption that the time scale for thermal fluctuations is slow compared to the shift in the dipole frequency caused by the fluctuations (adiabatic motion), the observed GDR strength function consists of a weighted average over all shapes and orientations. Projecting angular momentum, $J$, the GDR cross section is evaluated via [9]

$\sigma(E)=Z_{J}^{-1} \int \frac{\mathcal{D}[\alpha]}{\mathcal{I}(\beta, \gamma, \theta, \psi)^{3 / 2}} \sigma\left(\vec{\alpha}, \omega_{J} ; E\right) \mathrm{e}^{-F(T, \vec{\alpha}, J) / T}$,

where $E$ is the photon energy, $\mathcal{D}[\alpha]=\beta^{4} d \beta \sin (3 \gamma) d \gamma \sin \theta d \theta d \phi d \psi$ is the volume element, $Z_{J}=\int \mathcal{D}[\alpha] / \mathcal{I}^{3 / 2} \mathrm{e}^{-F / T}$, and the factor $\mathcal{I}(\beta, \gamma, \theta, \psi)$ is given by

$\mathcal{I}(\beta, \gamma, \theta, \psi)=I_{1} \cos ^{2} \psi \sin ^{2} \theta+I_{2} \sin ^{2} \psi \sin ^{2} \theta+I_{3} \cos ^{2} \theta$,

where the $I_{k}$ represent the deformation-dependent principal moments of inertia. Here, rigid-body values for the moments of inertia were used assuming the radius to be $R=$ $1.2 A^{1 / 3} \mathrm{fm}$. The free energy is given by

$F(T, \vec{\alpha}, J)=F\left(T, \vec{\alpha}, \omega_{\text {rot }}=0\right)+(J+1 / 2)^{2} / 2 \mathcal{I}(\beta, \gamma, \theta, \psi)$,

where $F\left(T, \vec{\alpha}, \omega_{\text {rot }}=0\right)$ is the free energy evaluated in the cranking approximation with rotational frequency, $\omega_{\text {rot }}$, equal to zero. Finally, the GDR cross section $\sigma\left(\vec{\alpha}, \omega_{J} ; E\right)$ is evaluated at the saddle-point frequency $\omega_{J}=(J+1 / 2) / \mathcal{I}(\beta, \gamma, \theta, \psi)$ (see below). 
Here, the GDR is modeled with a rotating, three-dimensional harmonic oscillator composed of three fundamental modes whose energies are given by [10]

$E_{k}=E_{0} \exp \left[-\sqrt{\frac{5}{4 \pi}} \beta \cos \left(\gamma+\frac{2 \pi k}{3}\right)\right]$,

where $E_{0} \approx 70 A^{-1 / 3}$ is the centroid energy for the spherical shape. Including the Coriolis term, the Hamiltonian for the GDR in the intrinsic frame may be written as [11]

$H_{D}=\sum_{k}\left(p_{k}^{2}+E_{k}^{2} d_{k}^{2}\right)-\vec{\omega}_{r o t} \cdot(\vec{d} \times \vec{p})$

where $d_{k}$ and $p_{k}$ are the coordinates and conjugate momenta associated with the dipole vibration and $\vec{\omega}_{\text {rot }}=\omega_{\text {rot }} \hat{z}$ is the rotational frequency. The GDR cross section is evaluated with the eigenstates $|\nu\rangle$ of $H_{D}$ and may be written as

$\sigma(E)=\sigma_{0} \sum_{\mu, \nu}\left|\left\langle\nu\left|d_{\mu}\right| 0\right\rangle\right|^{2} E\left[B W\left(E, E_{\nu}, \Gamma_{\nu}\right)-B W\left(E,-E_{\nu}, \Gamma_{\nu}\right)\right]$

where $\sigma_{0}=\left(4 \pi^{2} e^{2} \hbar / 3 m c\right)(2 Z N / A), \mu$ denotes the spherical components of the dipole, $B W\left(E, E^{\prime}, \Gamma\right)=\Gamma /\left[2 \pi\left(\left(E-E^{\prime}\right)^{2}+\Gamma^{2} / 4\right)\right]$, and $\Gamma_{\nu}$ is the intrinsic damping width for the resonance. In keeping with experimental findings [12], $\Gamma_{\nu}$ depends on the centroid energy $E_{\nu}$ via $\Gamma_{\nu}=\Gamma_{0}\left(E_{\nu} / E_{0}\right)^{\delta}$, where $\Gamma_{0}$ is the width for the spherical shape and $\delta \approx 1.8$. The laboratory cross section is evaluated by rotating the matrix elements $\left\langle\nu\left|d_{\mu}\right| 0\right\rangle$ from the intrinsic frame to the fixed external reference frame and by shifting the dipole energies associated with the intrinsic $\mu$ components by $-\mu \omega_{\text {rot }}$ [11]. Here, the parameters $E_{0}$ and $\Gamma_{0}$ were taken from ground-state data and are $E_{0}=15.2 \mathrm{MeV}$ and $\Gamma_{0}=5.0 \mathrm{MeV}$ for ${ }^{120} \mathrm{Sn}$ and $E_{0}=3.8 \mathrm{MeV}$ and $\Gamma_{0}=4.0 \mathrm{MeV}$ for ${ }^{208} \mathrm{~Pb}$, respectively.

The nuclear free energies were computed using the standard Nilsson-Strutinsky [13] procedure extended to finite temperature [14] using the Nilsson and liquid-drop parameters of Refs. [15] and [16], respectively. For the most part, the shell corrections for ${ }^{120} \mathrm{Sn}$ were found to be quite small, and for all practical purposes can be ignored. This is in sharp contrast to ${ }^{208} \mathrm{~Pb}$, where, at low temperatures, strong shell corrections $(\sim-15 \mathrm{MeV}$ at $T=0 \mathrm{MeV}$ ) are found that favor the spherical shape. Given the large difference between the free energies it is reasonable to expect that the strong spherical shell corrections in ${ }^{208} \mathrm{~Pb}$ will lead to a suppression of the FWHM at lower temperatures.

\section{RESULTS}

Shown in Fig. 1 are the results obtained for the FWHM of the GDR strength function for ${ }^{120} \mathrm{Sn}$ and ${ }^{208} \mathrm{~Pb}$ as a function of temperature in comparsion with experimental data [8] (filled circles). The solid line represents the theoretical values obtained with zero angular momentum. To illustrate the dependence on angular momentum, the FWHM for ${ }^{120} \mathrm{Sn}$ at $T=3.0 \mathrm{MeV}$ is plotted as a function of angular momentum in Fig. 2, where it is seen that for $J \leq 25 \hbar$ the FWHM is essentially unchanged from the $J=0 \hbar$ value. The effects on the FWHM in ${ }^{208} \mathrm{~Pb}$ due to angular momentum are even less pronounced than in the case for ${ }^{120} \mathrm{Sn}$ because of the larger moment of inertia. Note that the largest average angular 


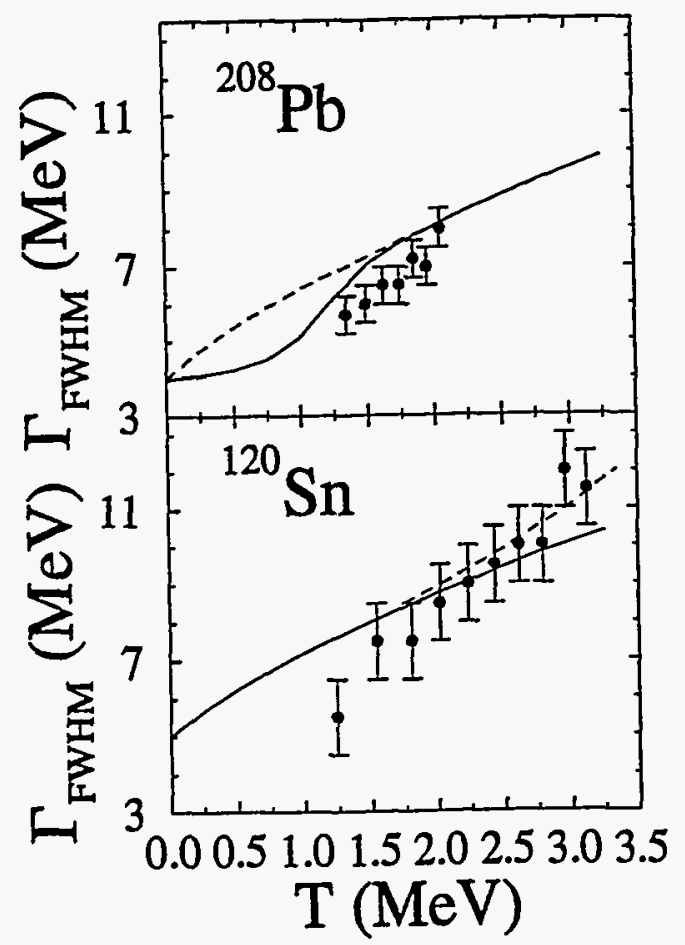

Figure 1. Comparison between theoretical (solid lines) and experimental (filled circles) values of the FWHM for ${ }^{120} \mathrm{Sn}$ and ${ }^{208} \mathrm{~Pb}$. For ${ }^{208} \mathrm{~Pb}$, the dashed line is the FWHM obtained assuming no shell corrections. For ${ }^{120} \mathrm{Sn}$, the dashed line represents the FWHM including $\Gamma_{c n}$.

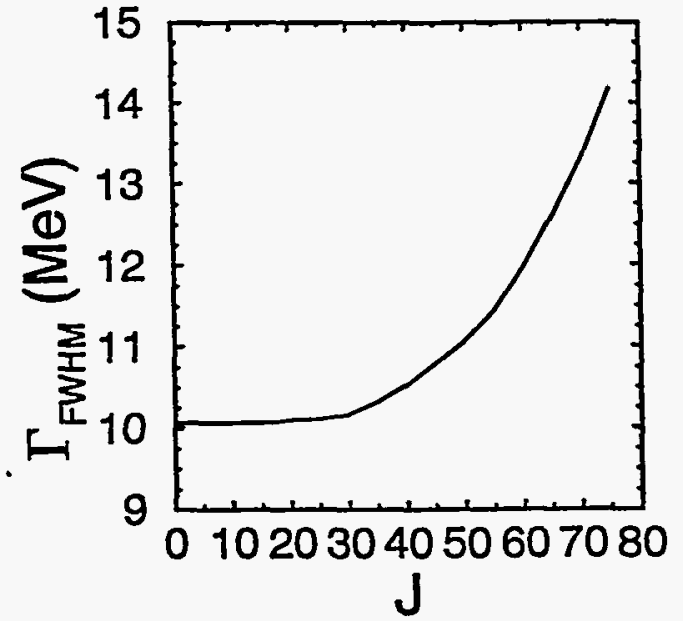

Figure 2. The FWHM for ${ }^{120} \mathrm{Sn}$ at $T=$ $3.0 \mathrm{MeV}$ as a function of angular momentum.

momentum in the systems studied experimentally is of the order $20 \hbar$ [8], and, therefore, the effects due to angular momentum are expected to be negligible.

To be noted in Fig. 1 is the overall agreement between theory and experiment. In particular, the dependence in the FWHM on temperature is different between ${ }^{120} \mathrm{Sn}$ and ${ }^{208} \mathrm{~Pb}$. The FWHM in ${ }^{208} \mathrm{~Pb}$ appears to be suppressed somewhat at lower temperatures relative to ${ }^{120} \mathrm{Sn}$. This is a feature that can be attributed to the rather strong shell corrections in ${ }^{208} \mathrm{~Pb}$ that favor the spherical shape at low temperatures. The effect of such strong shell corrections is to limit the effects of thermal fluctuations at low temperatures, thereby reducing the observed width. This is illustrated in Fig. 1, where the dashed line in the panel for ${ }^{208} \mathrm{~Pb}$ indicates the FWHM assuming no shell corrections.

Finally, we note some slight discrepancies between the adiabatic model and experiment for ${ }^{120} \mathrm{Sn}$ at the highest temperatures. At $T \approx 2.8-3.1 \mathrm{MeV}$, the experimental FWHM is somewhat larger than the theoretical values, and may indicate a systematic trend to be observed at higher temperatures. Shown in Fig. 3 is the FWHM for ${ }^{120} \mathrm{Sn}$ at $T=3.12 \mathrm{MeV}$ 


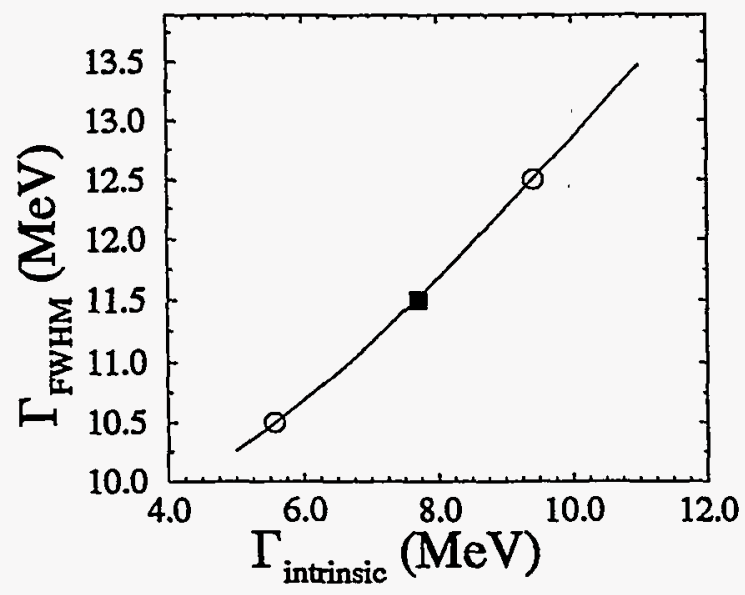

Figure 3. The FWHM for ${ }^{120} \mathrm{Sn}$ at $T=3.12 \mathrm{MeV}$ as a function of the intrinsic width $\Gamma_{0}$ (solid line). The experimental value of $11.5 \pm 1.0 \mathrm{MeV}$ is represented by the filled square $(11.5 \mathrm{MeV})$ and the open circles $( \pm 1 \mathrm{MeV})$.

as a function of the intrinsic width $\Gamma_{0}$. At this temperature, the experimental FWHM is $11.5 \pm 1.0 \mathrm{MeV}$, and we may infer from this data a value of $\Gamma_{0}=7.7_{-2.1}^{+1.7} \mathrm{MeV}$, as indicated by the solid square (FWHM=11.5 MeV) and open circles $( \pm 1 \mathrm{MeV}$ ) in Fig. 3 . We note that this is consistent with the concept that the width observed for the GDR in hot nuclei should be increased because of the evaporation of particles from the compound nucleus [6]. At higher excitation energies, the decay rate for particle evaporation increases, and because of the uncertainty principle, the energy of the emitted GDR photon cannot be known with a precision better than $\Gamma_{c n}=\Gamma_{e v}^{b e f o r e}+\Gamma_{e v}^{a f t e r}$, where $\Gamma_{e v}^{b e f o r e(a f t e r)}$ is the width for particle evaporation before and after the emission of the GDR photon. As such, the intrinsic widths of the GDR strength function are increased via $\Gamma_{\nu}^{\prime} \rightarrow \Gamma_{\nu}+\Gamma_{c n}$. To estimate $\Gamma_{c n}$ we refer to Fig. 2 of ref. [5], where $\Gamma_{e v}$ is plotted as a function of excitation energy. At a temperature $T \approx 3.1 \mathrm{Mev}\left(E_{x} \approx 130-150 \mathrm{MeV}\right)$, we deduce $\Gamma_{c n} \approx 2.1 \mathrm{MeV}$, which is in good agreement with the experimental results as is illustrated in Fig. 3 . In addition, we have computed the FWHM for ${ }^{120} \mathrm{Sn}$ as a function of temperature including $\Gamma_{c n}$. The resulting values are plotted in Fig. 1, with the dashed line, and give better overall agreement with experiment.

\section{CONCLUSIONS}

We conclude by noting that a systematic study of the FWHM of the GDR as a function of temperature for the nuclei ${ }^{120} \mathrm{Sn}$ and ${ }^{208} \mathrm{~Pb}$ confirms the overall theoretical picture of the GDR in hot nuclei; in particular, the role played by large-amplitude thermal fluctuations of the nuclear shape. This is confirmed by the good agreement between theory and experiment achieved over a range of temperatures from 1.25-3.2 MeV and by the 
differences in the behavior of the FWHM for ${ }^{120} \mathrm{Sn}$ and ${ }^{208} \mathrm{~Pb}$, which can be attributed to the presence of strong shell corrections favoring spherical shapes in ${ }^{208} \mathrm{~Pb}$ that are absent in ${ }^{120} \mathrm{Sn}$. Finally, the increase in the FWHM over that expected from thermal averaging at temperatures of the order $3.0 \mathrm{MeV}$ is in accordance with the increase expected from the particle evaporation of the compound system.

\section{ACKNOWLEDGMENTS}

Theoretical nuclear physics research at the University Tennessee is supported by the U.S. Department of Energy through contract DE-FG05-93ER40770. Oak Ridge National Laboratory is managed for the U.S. Department of Energy by Lockheed Martin Energy Systems, Inc. under contract No. DE-AC05-840R21400.

\section{REFERENCES}

1. K.A. Snover, Annu. Rev. Nucl. part. Sci. 36, 545 (1986); J.J. Gaardhøje, Annu. Rev. Nucl. Part. Sci. 42, 483 (1992).

2. P.F. Bortignon et al., Nucl. Phys. A460, 149 (1986); F.V. De Blasio et al., Phys. Rev. Lett. 68, 1663 (1992).

3. M. Gallardo et al., Nucl. Phys. A443, 415 (1985); Y. Alhassid, B. Bush and S. Levit, Phys. Rev. Lett. 61, 294 (1988); J.M. Pacheco, C. Yannouleas, and R.A. Broglia, Phys. Rev. Lett. 61, 1926 (1988); Y. Alhassid and B. Bush, Nucl. Phys. A509, 461 (1990).

4. B. Lauritzen et al., Phys. Lett. B207, 238 (1988); Y. Alhassid and B. Bush, Phys. Rev. Lett. 63, 2452 (1989); W.E. Ormand et al., Phys. Rev. Lett. 64, 2254 (1990); W.E. Ormand et al., Phys. Rev. Lett, 69, 2905 (1992).

5. P.F. Bortignon, A. Bracco, D. Brink, and R.A. Broglia, Phys. Rev. Lett. 67, 3360 (1991).

6. Ph. Chomaz, Phys. Lett. B347, 1 (1995).

7. A. Bracco et al., Phys. Rev. Lett. 74, 3748 (1995); A. Bracco et al., Proc. of the Groningen Conference on Giant Resonances, 28 June - 1 July, 1995, to be published in Nucl. Phys. A.

8. E. Ramakrishnan et al., Proc. of the Groningen Conference on Giant Resonances, 28 June - 1 July, 1995, to be published in Nucl. Phys. A; E. Ramakrishnan et al., submitted to Phys. Rev. Lett.

9. Y. Alhassid and N. Whelan, Nucl. Phys. A565, 427 (1993); W.E. Ormand, P.F. Bortignon, R.A. Broglia, to be published.

10. A. Bohr and B.R. Mottelson, Nuclear Structure (Benjamin, Reading, MA, 1975), Vol. II.

11. K. Neergård, Phys. Lett. 110B, 7 (1982).

12. P. Carlos et al., Nucl. Phys. A219, 61 (1974).

13. V.M. Strutinsky, Yad. Fiz. 3, 614 (1966) [Sov. J. Nucl. Phys. 3, 449 (1966)]; Ark. Fys. 36, 629 (1966); Nucl. Phys. A95, 420 (1967); Nucl. Phys. A122, 1 (1968).

14. M. Brack and P. Quentin, Nucl. Phys. A361, 35 (1981).

15. S.G. Nilsson et al., Nucl. Phys. A131, 1 (1969).

16. C. Guet et al., Phys. Lett. B205, 427 (1988). 
